\title{
Une problématique nouvelle
}

«Avenir de la formation et école de l'avenir » en Allemagne

A new issue. The "Future of training and the school of the future »: the German perspective

Una problemetica nueva. "Porvenir de la formation y escuela del parvenir » en Alemana.

\section{Dominique Gelin}

\section{OpenEdition Journals}

Édition électronique

URL : http://journals.openedition.org/ries/2796

DOI : 10.4000/ries.2796

ISSN : 2261-4265

\section{Éditeur}

Centre international d'études pédagogiques

\section{Édition imprimée}

Date de publication : 1 mars 1999

Pagination : 71-75

ISBN : 9771254459005

ISSN : $1254-4590$

\section{Référence électronique}

Dominique Gelin, «Une problématique nouvelle », Revue internationale d'éducation de Sèvres [En ligne], 21 | mars 1999, mis en ligne le 16 avril 2013, consulté le 08 janvier 2020. URL : http:// journals.openedition.org/ries/2796 ; DOI : 10.4000/ries.2796

Ce document a été généré automatiquement le 8 janvier 2020

(c) Tous droits réservés 


\title{
Une problématique nouvelle
}

\author{
«Avenir de la formation et école de l'avenir » en Allemagne \\ A new issue. The "Future of training and the school of the future »: the German \\ perspective \\ Una problemetica nueva. «Porvenir de la formation y escuela del parvenir » en \\ Alemana.
}

Dominique Gelin

1 Une commission mise en place en 1992 en Rhénanie-Westphalie sur le thème " Avenir de la formation, école de l'avenir » a abouti en 1995 à un document fondateur auquel toutes les commissions qui ont siégé entre temps dans les autres Länder sur le thème de la formation des enseignants font maintenant référence. Ce texte qui brosse le cadre de l'évolution nécessaire de l'école dans une société en profonde mutation s'efforce de définir une professionnalité enseignante(en terme de compétences), pointe les déficits de la formation actuelle et en tire des conclusions pour une nécessaire réforme de la formation des enseignants.

2 Tous ces points ont été repris et développés dans les documents élaborés par la Société allemande pour les sciences de l'éducation au sein d'une commission «Pédagogie et formation des enseignants " (juin 1997) et à l'occasion de la table ronde « formation des enseignants » de la Sarre (juin 1997).

3 Tous ces documents mettent en évidence l'investissement personnel des enseignants en exercice actuellement, ce qui ne permet pas, cependant, de toujours faire face aux difficultés nouvelles et peut de ce fait entraîner un grand découragement. Cette approche peut apparaître féconde pour l'avenir: il s'agit de former de vrais professionnels, capables de réagir aux mutations, conscients de ne pas être formés une fois pour toutes et convaincus que la formation est un processus auquel ils doivent participer tout au long de leur vie professionnelle. 


\section{La formation : un processus continu}

4 Dans cette perspective, les futurs enseignants devront acquérir une compétence disciplinaire assez approfondie et réfléchie pour être à même d'envisager les problèmes de la transposition didactique et de voir les liens avec d'autres champs disciplinaires. Ils devront percevoir clairement leur propre rapport à cette discipline, au métier et aux élèves, être capables d'évaluer leur action et de la faire évoluer, de mettre en place des situations d'apprentissage adaptées à la diversité des élèves, de travailler en équipes, en collaboration avec les parents d'élèves et d'autres partenaires sociaux, d'analyser et de réagir positivement à la complexité des situations à l'intérieur du système scolaire.

Pour aider les enseignants à envisager la formation comme un processus continu qui accompagne la vie professionnelle, une réforme des structures et des modalités de la formation est nécessaire afin :

- de rendre plus lisibles, voire de faire vivre, les liens entre les trois phases de la formation (cursus universitaire initial, formation pratique et formation continue);

- de considérer qu'il ne s'agit là que des différentes phases d'un seul et même processus ;

- d'introduire, dès la première phase, une expérience pratique supervisée par les universitaires et les praticiens et d'en faire un objet d'étude ;

- de faire en sorte que les modalités de cette formation (méthodes pédagogiques variées à tous les niveaux, interdisciplinarité, travail en équipes, coopération, partenariat...) soient une réelle propédeutique pour le métier d'enseignant, un champ d'observation et de recherche, une sorte de laboratoire pédagogique ;

- de faire participer les futurs enseignants dès les premiers semestres de leur formation à des travaux de recherche dans les champs épistémologique, pédagogique, didactique ou sociologique, afin de leur donner les outils nécessaires pour analyser les objets de savoir, leur propre action et le système éducatif qui doivent être objets d'étude tout autant que les savoirs à enseigner ;

- de créer au sein des universités, en très étroite collaboration avec ces dernières, des lieux spécifiques de formation pour les enseignants qui devraient institutionnaliser et organiser les rapprochements entre les sciences de l'éducation, l'enseignement des disciplines, la didactique et la réflexion sur la pratique, mettre chacun de ces domaines au service des trois autres, de permettre une meilleure articulation des trois phases de la formation, une meilleure collaboration des différents personnels intervenant dans la formation (universitaires, didacticiens-praticiens, intervenants $\mathrm{du}$ monde social), d'aider au développement d'une recherche appliquée et créer les bases d'une professionnalité ;

- d'institutionnaliser l'accompagnement lors de l'année de prise de fonction et de marquer ainsi que l'établissement scolaire doit devenir le lieu privilégié d'une formation qui ne fait que commencer.

\section{L'université gage de formation de haut niveau}

$6 \quad$ Tous ces textes et projets insistent sur la place essentielle de l'université dans toutes les phases du processus de formation et sur l'importance d'une formation de haut niveau, et plaident pour le maintien de la formation à l'université (certains Länder envisageaient de transférer la formation des enseignants dans les Fachhochschulen équivalents des IUT). Ils ne cachent pas néanmoins que le rôle dans lequel l'université se confine actuellement, à savoir la seule qualification « scientifique » - l'enseignement 
des savoirs savants - sans en envisager la transposition didactique et les modes de transmission, le cloisonnement des différents départements, la relégation des domaines transdisciplinaires, le manque de porosité avec l'école et ses acteurs expliquent, en partie, les dysfonctionnements actuels. Les futurs enseignants allemands ont certes des cours de psychologie, de sociologie et de sciences de l'éducation, mais ces cours sont relativement en "apesanteur", ils ne s'appuient pas sur l'expérience des futurs enseignants, domaine d'investigation de la deuxième phase de la formation, réservée aux didacticiens-praticiens qui ont très peu de relations avec l'université.

7 Le nécessaire développement de la recherche en éducation (didactique des disciplines, sciences de l'éducation...), parent pauvre jusqu'à ce jour, voire considérée comme de la sous-recherche, l'initiation et la participation à cette recherche, à toutes les phases de la formation, la possibilité pour des enseignants plus expérimentés de s'engager, pour une part de leur service, dans des recherches qui ne portent pas uniquement sur des objets de savoir pointus, mais sur la transmission des savoirs, les phénomènes d'apprentissage ou l'organisation de l'école, sont considérés comme un moteur essentiel du changement à venir et de la professionnalisation.

8 Il y a derrière ces projets de réforme, dont certains ont déjà abouti, une conception nouvelle du rôle de la formation universitaire et de la recherche qui consiste à :

- envisager le savoir et la qualification universitaire, non pas en soi et pour soi, mais en l'inscrivant dans une perspective de réflexion, de recherche et de transmission en même temps ;

- faire de la pratique et de son lieu d'exercice (l'établissement scolaire) un objet d'observation, d'enseignement et de recherche et, pour cela, collaborer étroitement avec les praticiens qui, eux aussi, devront bouger et accepter que le «terrain » ne soit pas leur domaine réservé ;

- contraindre l'enseignement universitaire à s'intéresser aux méthodes pédagogiques, non plus comme objet d'études, mais dans la pratique des cours et à s'ouvrir à l'interdisciplinarité.

\section{Quelles évolutions?}

Les concepteurs de ces réformes pensent que ces évolutions seront facilitées par la mise en place d'«instituts universitaires de formation" dont les missions rappellent fortement celles des IUFM français depuis que la formation continue leur a été déléguée. Ils y voient la possibilité de faire travailler ensemble des "formateurs » d'horizons très différents, avec un objet commun, au sein de l'université, mais sont très conscients du fait qu'il s'agit là d'un " pari » qui ne saurait aboutir sans une pression forte du politique.

10 On retrouve dans tous ces projets des questions qui agitent, en France, le monde de la formation : « lier étroitement formation initiale et formation continue, mieux inscrire ainsi dans le temps l'acquisition progressive des compétences professionnelles complexes et multiples requises pour l'exercice du métier, renforcer la dimension universitaire de la formation continue », lit-on dans la lettre sur la formation adressée par le ministre de l'Éducation nationale aux recteurs.

11 L'Allemagne semble avoir quelques longueurs d'avance, les projets ont déjà fait l'objet de débats entre les différentes parties concernées et on sent cependant en arrière-plan, comme en France, la défense de certains intérêts catégoriels (universitaires spécialistes 
des disciplines/sciences de l'éducation, universitaires/praticiens), des combats idéologiques (l'enseignant instructeur/l'enseignant éducateur) et une grande vigilance par rapport aux responsables politiques qu'on soupçonne, souvent à juste titre, de vouloir faire, sous couvert de réformes, des économies partout où c'est possible (projet de délégation de la formation des enseignants sarrois à l'université du Palatinat, abandonné entre temps, par exemple; suppression d'un semestre de stage pratique accompagné, justifié par l'introduction de stages pratiques dès la phase initiale...).

Il est intéressant de voir que les Allemands qui, aux yeux des Français, ont une conception moins encyclopédique de l'enseignement mettent l'accent sur la nécessité d'une formation universitaire de haut niveau et voient dans l'initiation et la participation des praticiens à la recherche une des clefs pour l'avenir. Mais, on l'a vu, il s'agira alors d'un enseignement universitaire pour le moins "rénové ", capable de prendre en compte la pratique, d'interroger la pertinence des savoirs enseignés et d'envisager, dès l'université, les modalités concrètes de la transmission. De la même façon, la conception sous-jacente de la recherche implique une réelle interpénétration recherche/pratique, tant par le choix des terrains de recherche que par le recrutement des chercheurs. On retrouve là encore une problématique française actuelle: «La recherche sur l'école ne peut pas s'appuyer sur la seule science des laboratoires, elle doit lier cette science au travail des acteurs de l'école. Les IUFM doivent jouer un rôle essentiel dans ce domaine ${ }^{1} »$.

\section{NOTES}

1. François Dubet, in : XXI esiècle, $\mathrm{n}^{\circ} 2, \mathrm{p} .23$.

\section{RÉSUMÉS}

Une réflexion générale sur la formation des enseignants a été menée ces derniers temps dans de nombreux Länder allemands à partir d'une étude menée en Rhénanie-Westphalie à ce sujet, en 1995. Deux thèmes : la formation comme processus continu, le rôle de l'université comme garant d'une formation de haut niveau constituent les points forts de ce document de référence intitulé «Avenir de la formation, école de l'avenir ».

A general reflection on teacher training has been undertaken recently in many German Lànder from a study carried out in Rhénanie-Westphalie on this subject in 1995. Two themes: training as a continuous process and the role of the university as a guarantee of a high level of training are the main points in this reference document entitled « The future of training, the school of the future ». 
Ultimamente, se llevó a cabo una reflexión general a propósito de la for-mación de los docentes en numerosos Länder alemanes a partir de un estudio hecho en Renania-Westfalia en 1995. Dos temas : la formación como proceso permanente y el papel de la universidad en cuanto fiador de una formación de alto nivel constituyen los puntos dominantes de este documento de consulta que se titula « Porvenir de la formaciôn, escuela del porvenir ».

\section{INDEX}

Index géographique : Allemagne

Mots-clés : enseignant, formation des enseignants, réforme de l'enseignement, université

\section{AUTEUR}

DOMINIQUE GELIN

Professeur, IUFM de Créteil (France) 